\title{
PREÁMBULO A LA JUSTIFICACIÓN DE LA FILOSOFÍA COMO CIENCIA
}

Nos proponemos desarrollar en este ensayo uno de los temas que exponen de manera más elocuente la problemática filosófica, o cuando menos un aspecto sui generis de dicha problemática, el cual promueve una polémica sin paralelo en ninguna otra disciplina del pensamiento: la posibilidad de considerarla como ciencia. El carácter peculiar de la cuestión -aunque no el más lógico- podría consistir en haberla ubicado alternativamente a niveles tan dispares como son el de la ciencia, por una parte, de una posible infraciencia, por otra, y de una hipotética superciencia, por una tercera.

Todo ello se ha dicho y reafirmado acerca de esta disciplina, en la cual se pone de relieve, mediante una exégesis histórica de sus doctrinas, que siempre han existido los tres niveles mencionados, aunque en distinto grado de evolución. Participan ellos de una referencia genérica a todas las formas del pensamiento, confrontada a la aplicabilidad específica que corresponde a las doctrinas filosóficas, en cuya virtud sería posible ensayar una clasificación de tales doctrinas a partir del criterio esgrimido en el distingo de los tres supuestos niveles epistémicos: la ciencia, la infraciencia y la superciencia; ellos comprenden los grandes sectores en que pueden agruparse las posturas habituales de la filosofía, lo cual no es óbice para que, en medio de semejante diversidad doxográfica, exista el común acuerdo de titularlas con el nombre de filosofia.

Señalaremos en qué consiste la situación peculiarisima. Históricamente, a la filosofía se ha atribuido, de un lado, una especie de carácter supracientífico al considerarla como reina de las ciencias, matriz fecunda y generosa de todo conocimiento, capaz de llegar al saber absoluto y revelar los abscónditos secretos de la naturaleza en sus causas primeras y fines últimos, propósito éste que haría sonrojar a cualquier cientifico que tuviera una aceptable conciencia limitativa de su tarea. Por otro lado, la filosofía está acusada de violar las reglas de la lógica que ella misma postula, incurriendo en vicios tan incongruentes como sería la pretensión de adquirir un conocimiento absoluto e interferir lo que afirma el pensamiento científico en torno a la experiencia. La frecuente imprecisión de sus objetivos pone en entredicho su existencia, de modo que el problema concerniente a la validez filosófica, contemplado desde el ángulo primario de su objetividad, no deja de ser inquietante. 
El ensayo que abordamos ahora corresponde a la realidad intrínseca de las posturas cuya abigarrada divergencia exige un análisis casuístico donde se compruebe la existencia de doctrinas que podrían incorporarse a cualquiera de las subdivisiones en que se manifiestan, o quizá a varias de ellas, habida cuenta de la abrumadora diversidad tética, metodológica y sistemática que se encuentra en las mismas. Nuestro propósito se limitará a ensayar la presentación general del problema a partir del planteamiento medular que requiere las condiciones básicas para constituir a la filosofía como ciencia; en caso contrario permanecerá esta disciplina a nivel de infraciencia, lo cual incluye la especulación peculiar de creer que puede erigirse en una especie de superciencia, como en efecto se ha creído repetidamente, e inclusive se piensa todavía en algunas ocasiones. Empero, la cuestión medular que se impone como norma de principio en este ensayo, radica en determinar la posibilidad básica que asiste a nuestra ocupación para justificarse como ciencia.

A tal efecto, uno de los problemas que definen de mejor manera el estado actual de la filosofía se encuentra en la dualidad antagónica y dialéctica establecida entre el antiguo filosofar especulativo y el moderno filosofar científico. Este último corresponde a la tendencia general que se manifiesta actualmente para ubicar a todas las formas del pensamiento en el nivel científico de un saber objetivamente válido y, aunque en épocas recientes se agudizó esta demanda de cientificidad, fuerza es admitir que desde hace tiempo se pretende implantarla como norma insoslayable en la reflexión. El tratamiento sinóptico del tema nos remite el antecedente más amplio en la historia del pensamiento, consistente en promover la rflexión a base de puntos de vista particulares en las correspondientes derivaciones sistemáticas o parasistemáticas que reciben el nombre de posturas filosóficas. Se ha entendido tradicionalmente por postura la actitud personal, o cuando menos particular, en que se maneja un criterio más o menos subjetivo para debatir los problemas. Dicho criterio es distinto en cada caso y para cada postura constituye uno de los muchos que pueden aplicarse al ejercicio filosófico.

Un enfoque directamente abocado a la crítica es el que toma como punto de partida la existencia indiscriminada de las posturas particulares, en lo cual es necesario resaltar como primera medida la incongruencia de su particularismo. Pero en este caso, como en otros, se presentan grados y matices; el extremo insostenible a que puede llegar cualquier teoría es el desenlace netamente subjetivo y estriba en la actitud personalista de quien afirma: yo creo, yo pienso, yo opino, como si fuera éste el argumento supremo de la reflexión. Al multiplicarse tal actitud, da cauce a la abigarrada pluralidad doxográfica que suele figurar como prototipo de la reflexión. EI análisis doctrinario demuestra que ésta es la antítesis de la cientificidad, pues resulta frecuente que cada quien opine como cree, no como sabe. El hecho irrevocable de las posturas, tal como se han manifestado hasta ahora, induce a cues- 
tionar severamente la cientificidad de nuestra disciplina, como ha sido cuestionada con frecuencia en el nódulo de sus más críticas disquisiciones.

Frente a tan controvertible realidad es necesario abrigar la convicción de que no pueden subsistir por más tiempo las posturas filosóficas entendidas a la antigua manera, como expresión particular y subjetiva; sus frecuentes contradicciones aparecen en términos contingentes sobre la aleatoria trayectoria de la cual se está queriendo liberar para ubicarse en un concepto univoco y definido, esto es, científico, que elimine la multiforme concurrencia doctrinaria, propendiendo en cambio a constituir la filosofía como ciencia. Por ello, la corrección que se impone al subjetivismo particular de las posturas señala un punto concluyente: la filosofía debe alejarse cada vez más de la constelación doxográfica para intentar la conversión del viejo filosofarpor-posturas al congruente filosofar-por-sistemas, erigiendo la postura personal en impersonal y la doctrina profesada en sistema demostrado. Sin embargo, todavía. la investigación se encuentra íntimamente ligada a las posturas particulares, de donde, al explicar su origen y consecuencias, llegamos a comprender la necesidad de que subsista un solo criterio como instrumento eficiente de la pesquisa. ¿Cómo puede realizarse el proyecto de erigir a la filosofía como ciencia, si no lo fuera, o de justificarla como tal, si ya lo hubiera sido?

Un primer paso consistirá en el examen de las relaciones que establece la filosofía con las ciencias particulares, o quizá de la relación específica donde encarna un aspecto muy importante del problema que nos ocupa; se trata del proceso que nosotros llamamos metátesis aporética y consiste en la progresiva transferencia que lleva a cabo la primera al adjudicar su problemática a las segundas; son éstas las que enajenan sus motivos de reflexión para convertirlos en objeto de estudio asequible por medio de la metodologia científica. El nombre de metátesis que otorgamos a este proceso significa transferencia de tesis, o sea la adjudicación aporética que acabamos de señalar. El proceso metatético, cuando no es consciente, y por lo mismo no se efectúa sobre una base metodológica común, auspicia en gran medida la confusión tantas veces señalada entre filosofía y ciencias, aunque, observado desde otro ángulo permite su mutua comprensión. Todo ello nos pone en camino de justificar a la filosoffa como ciencia, pues difícilmente se podría lograr este propósito dejando incólume la abundante gama de doctrinas contradictorias que constituyen la expresión casufstica de la indiscriminada doxografía subjetiva, en vez de lo cual es necesario proceder a su justificación dialéctica en el seno de la historia y a su reducción sistemática en los términos estructurales que establece su propia funcionalidad; al señalar el origen de las posturas y su constante proliferación, contemplamos el camino para reducirlas a unidad. Este es el punto de partida indispensable a nuestro propósito.

Lo que interesa a tal respecto es subrayar que del planteamiento autó- 
nomo señalado en la metátesis aporética depende la posibilidad de constituir a la filosofía como ciencia. Cumple en primer término el requisito elemental de exhibir un problema exclusivo; en caso contrario aparecerá una vez más la reiterada crisis que se contempla en el transcurso de la historia, cuya función ha consistido en mostrar a la filosofía como matriz de otras disciplinas, las cuales surgen de ella, pero se independizan al delimitar como propio un problema que al principio se había considerado filosófico pero, circunscrito a los alcances de su planteamiento, transmuta un tema particular del cual se encarga la disciplina de la misma índole que, por ello mismo, es ciencia particular. Este procedimiento cuestiona en alto grado la posibilidad de que exista un problema de carácter material, específicamente atribuible a la filosofía, y que al mismo tiempo derive de la experiencia, cuyas grandes mutaciones históricas registran la pretensión común de producir un concepto universal del mundo y de la vida, en contraste con el alcance circunscrito del conocimiento científico que, a pesar de los numerosos intentos en contrario, no trasciende el ámbito particular que necesariamente impone la experiencia.

La lección del pasado es muy elocuente en lo relativo al constante surgimiento de doctrinas escudadas en la mampara de una postura, o sea de un aparente sistema asumido en forma unilateral, frente a las demás que coexisten con ella en el panorama historiogénico de las ideas. Se trata de un testimonio fehaciente del carácter precientífico que ha distinguido a nuestra disciplina, habida cuenta de las contradicciones constantemente manifiestas en sus doctrinas y de la forma insoluta como se presentan a la mirada del observador. Para comprobar esta tesis bastaria un cotejo de lo que sucede en las ciencias particulares, donde no existen posturas al modo como se entienden filosóficamente, como si cada una tuviera la razón absoluta y no existieran las contradicciones que en verdad se manifiestan como resultado de su limitación y su relativismo. Así vemos sucederse unas a otras las tesis racionalistas, realistas, idealistas, relativistas, criticistas, empiristas, etc., esgrimiendo cada una su idea peculiar, que a la postre resulta insostenible frente a las otras, lo cual genera el panorama de contradicciones que ha hecho suponer a la filosofía como el maremagnum de opiniones irreductibles donde nunca llega a sentarse un definido acuerdo.

El concepto de postura entra en crisis cuando se observa con la suficiente imparcialidad para eliminar las opiniones subjetivas que en ella se manifiestan, clarificando al mismo tiempo la razón implícita de su origen. Este criterio se ha expuesto con la intención de ubicar a la filosofía en un plano científico $o$ paracientífico, mas no podría afirmarse que tal propósito se hubiera convertido en satisfactoria realidad, aunque esté reconocido como urgencia de primer orden; la tónica general del filosofar-por-posturas estriba -cuando menos en el consenso tradicional_ en pronunciarse contra cual- 
quier intento de cientificidad. Filosofía y ciencia, se dice, son irreductibles; no es posible convertir la ciencia en filosofía ni la filosofía en ciencia. Este criterio va en paralelo a la particularidad de las doctrinas, enlazando el nódulo histórico del proceso metatético para concluir especificando sus relaciones con las ciencias y la necesidad de configurar la filosofía como ciencia.

La cuestión crucial en nuestro propósito consiste en explicar por qué la filosofía debe asumir la categoría científica, teniendo a la vista la gama de exigencias planteadas para acreditarla en el campo académico de acuerdo al sentido que asume actualmente como investigación; de manera más amplia, para justificar su propia existencia en medio de las crisis evolutivas que la aquejan constantemente. Dicho problema se ha debatido a través de una abundante exegética que aparece y reaparece en forma recurrente; se manifiesta en primer término mediante la puesta en crisis de las teorias formuladas de manera dogmática al estipular la conciencia crítica de la reflexión como tónica fundamental de la etapa culminante en cada perfodo, cuyo último nivel de madurez propende a generar la autoconciencia del método y el sistema. Con ello se eleva la problemática, a partir de una actitud dubitativa, a la preocupación medular que gira en torno a su validez y constituye de manera implícita o explícita la base irremplazable para conferir el carácter científico a la filosofía. Podríamos decir que la conciencia crítica de nuestro tiempo ha trafdo el saludable efecto de rechazar todo pretendido saber que, en aras de la especulación, no pudiera verificarse objetivamente; ésta es la base irremplazable en su erección científica.

De cualquier manera, no deja de ser inquietante que todavía en la actualidad se debata la vieja cuestión concerniente al tipo de ciencia que debe ser la filosofía, e incluso llega a manifestarse cierta renuencia a admitir en ella el carácter de cientificidad. Parecería que tal virtud, inherente a toda disciplina intelectiva, le está vedada por los requerimientos de fijar una problemática concreta, de obtener verdades relativas y comprobarlas en la experiencia, en vista de su tradicional pretensión de universalidad, absolutez y eternidad, que en sus más dogmáticos avatares ha caracterizado a la madre de todas las ciencias.

Esta clase de pretensiones, vivas aún en las polémicas del campo filosófico, resultarían anacrónicas si hipotéticamente se trasladaran a cualquiera de las ciencias particulares que se estabilizaron desde hace tiempo. La ingente preocupación en torno a la cientificidad filosófica obedece precisamente al hecho de no haberla logrado por completo, pese a los numerosos intentos realizados desde la época griega; pero no obstante el gran favor otorgado a este propósito, existen algunos impedimentos que obedecen a ciertas deficiencias expuestas de continuo en la reflexión; tal es, en primer término, el ir y venir de las posturas particulares, cuya más conspicua taxonomía se basa en el dualismo epistémico que escinde, conforme a los factores filoge- 
néticos del acto cognitivo, realismo e idealismo, empirismo y abstractismo, objetivismo y subjetivismo, vinculados en la síntesis dialéctica del proceso teorético e integrativo del saber.

La cuestión de la cientificidad es debatida - no siempre en forma expresa- a través de los siglos, mediante una problemática que figura como tónica de las posturas críticas en la etapa agógica de cada periodo y denota en cierto modo su más alto nivel en la conciencia crítica que propende a la relativización del saber obtenido en ese periodo. Con gran frecuencia las posturas dubitativas asumen una preocupación análoga al señalar la multiplicidad insoluta de las doctrinas y se erigen en críticas cuando aceptan la irreductibilidad de las tesis examinadas; como resultado de esta asunción crítica irrumpe la necesidad de trascender la abigarrada doxografía confiriendo a la reflexión un carácter cientifico mediante el acendramiento de sus virtudes sistemáticas. De cualquier modo, este hecho no se ha reconocido unívocamente, ni se ha planteado con la decisiva urgencia del caso; la necesidad de una rigurosa presuposición científica sigue en perspectiva a pesar de los numerosos intentos producidos desde la Antigüedad, cuando por vez primera se admitió la exigencia de acometer el pensamiento filosófico en paralelo al científico, e inclusive de asimilar el primero al segundo.

\section{II}

Procedamos ahora a plantear específicamente el problema que nos ocupa; nada mejor para ello que implantar un despeje definitorio. El concepto más general que pueda establecerse de una ciencia nos lleva a considerarla, en primer término, como un conjunto de conocimientos. Así, al mismo tiempo que se rechaza cualquier tipo de especulación se postula que todo conocimiento verdadero es científico y queda acreditado como autónomo mediante la definida circunscripción de su objeto.

Esta convicción parece elemental, y lo es en realidad como principio básico, mas no por ello fácil de cumplir; es necesario, en primer término, esclarecer las condiciones que satisfará toda ciencia y los errores que evitará, lo cual no está suficientemente resuelto a pesar de algunas preocupaciones externadas para discernir las circunstancias que orillan al error, y por consiguiente, a la negación del saber. Esta negación supone un arraigo en la subjetividad; sabemos que el conocimiento debe ser verificado lato sensu en la experiencia, donde se comprueba la objetividad de los enunciados. Aceptar el requisito universal de objetividad tiene como consecuencia la definitiva superación del impulso ficcional que describe Kant en la célebre Alegoria de la paloma, por cuya virtud el conocimiento, liberado de la sujeción a la experiencia, volaría mejor que sometido a la densa atmósfera de la realidad. Esto vale particularmente para el designio clásico de la filosofía; no obstante 
el considerable progreso realizado en el campo de la critificación doctrinaria, resulta inevitable replantear la vieja cuestión absolutista y romántica que demanda un concepto universal del mundo y de la vida, en cuya posibilidad surgen cada vez mayores dudas, sobre todo cuando la universalidad pretende obtenerse mediante la conversión hipostática de un elemento particular. No ha podido erradicarse la sugerente imagen universal que inspira la cosmopsicovisión, pero la crítica moderna circunscribe el pensamiento a los estrechos límites que comporta la definición estricta de cada objeto y su verificación en el sistema de conocimientos que origina.

A pesar de todo, la convicción en torno a la cientificidad filosófica se traduce en la generalizada tónica de nuestro tiempo para promover la nueva revolución en esta disciplina; semejante convicción se extiende a medida que el concepto mismo de ciencia se hace más dúctil y trasciende la acepción demasiado circunscrita que ha considerado como ciencia únicamente la exacta o rigurosa; por ello se vuelca la mirada en la acepción matematicista, de análoga manera a como sucedió en el siglo xvir, no para eliminar de ella a la filosofía, sino al contrario, para incluirla en su seno. En todo caso, la preocupación de cientificidad representa o debe representar el vértice común en el ámbito de la filosofía contemporánea, aceptando el requisito básico de no cohonestar la lucubración abstracta con la investigación filosófica.

Reconocemos, pues, que la filosoffa es o debe ser ciencia. Pero, ¿qué modelo de ciencia conviene a la filosofía? Para aclararlo es necesario acudir a lo que significa el concepto de ciencia. Tiene dos sentidos: uno amplio, que equivale genéricamente a conocimiento, y otro restringido, que conlleva especificamente la acepción de conociminto sistemático. Entrambos cumplen la condición primordial de enunciar una verdad; cualquiera de las expresiones predicativas que se llaman conocimientos, entraña una verdad que puede ser demostrada. Afirmamos entonces que: todo conocimiento, por el hecho de ser conocimiento, es demostrado, y por ser demostrado es verdadero, $y$ por ser verdadero es cientifico. Esta tesis se verifica a nivel elemental, adoptando como punto de partida los conocimientos derivados de la vida cotidiana y, a pesar de su carácter esporádico, pertenecen a una o varias ciencias. Lo más probable es que cada conocimiento incidental figure en calidad de motivación empírica: señalará un problema por determinar a la ciencia encargada de resolverlo.

Por ejemplo, si yo digo me duele la cabeza, estoy emitiendo un conocimiento verdadero que corresponde a un suceso real; aparentemente no es conocimiento científico, pero concierne al área específica de una problemática inherente a la medicina, cuya más amplia determinabilidad registra la explicación de por qué me duele la cabeza y también el precedimiento adecuado para prevenir o erradicar el dolor. Análogamente, una observación circunscrita como puede ser el verbo impersonal llueve, significa que se está 
produciendo la precipitación pluvial y constituye un problema para otra ciencia como es la meteorología, de modo que el conocimiento involucrado incidentalmente en la palabra llueve, es también un conocimiento cientifico en la medida que pertenece a una ciencia, donde figura como problema por determinar y ocupa el nivel más elemental de la investigación.

No es común admitir como configurativas de un sistema las expresiones aisladas, aunque encarnen una verdad; algunas de ellas llegan inclusive a reconocerse como axiomas del intelecto $\mathrm{y}$, sin embargo, observamos que cada uno de los fragmentos incidentales del saber pertenece a una ciencia. En ocasiones a más de una; por ejemplo, ciertas acotaciones cotidianas se incluirían en el saber antropológico, cuando se refieren a peculiaridades del ser humano; otras se clasificarian en el conocimiento físico si atañen a especifidades de la naturaleza; otras más involucran conceptos psicológicos cuando se ocupan del alma. Todo pensamiento, por más esporádico que se suponga, concierne de hecho a una o varias ciencias no obstante que, al margen de esta adjudicación, tales pensamientos quedarían en calidad de ideas efímeras, no científicas; aparentemente como producto de alguna observación incidental.

En el campo filosófico esta manera de discurrir se expone en fragmentos, opiniones, máximas o sentencias que constituyen efectivamente un estilo de reflexión alejado de las complejas formas sistemáticas del filosofar académico; pero ello no autoriza a calificarlo como falso o inoperante, si nos atenemos al más amplio designio del pensar, consistente en producir verdades; algunas de ellas llegan a ser de gran alcance y en cierto modo realizan el hito filo. sófico tendente a descubrir las verdades más profundas del intelecto y expresar la verdad radicalmente universal que concierne en cada caso al problema respectivo. De esta suerte, por radicales y universales, dichos fragmentos llegan a ser muy elocuentes, e inclusive ciertas doctrinas encuentran su máxima expresión en resumidos apotegmas de unas cuantas palabras, lo cual les ha hecho particularmente accesibles al gran público; por ello se encuentran en el repertorio de las consejas populares y son pensamientos de cuño corriente con un sinnúmero de aplicaciones en la vida cotidiana. Algunas constituyen verdades básicas del saber académico; decir que todo tiene un limite, expresa la gran verdad de que no hay nada infinito; un pensamiento análogio nos lleva a reconocer que todo es relativo, como contraparte de que nada es absoluto. El filosofar anecdótico se ha enriquecido mediante sentencias definitivamente inscritas en la posteridad con el crédito eponímico del autor. ¿Quién no ha escuchado alguna vez el apotegma socrático: Sólo sé que no sé nada? ¿Podría ignorarse que Descartes inmortalizó el reconocido entimema: Pienso, luego existo? $\mathrm{Y}$ así sucesivamente.

Concluimos entonces que el concepto lato de ciencia obliga a acreditarse como conocimiento verdadero, lo cual no sucede en forma inequívoca; existen 
óbices cuya interferencia en el proceso epistemológico impide la consecución incontrovertible de la verdad, como se verifica en todo el campo del saber mediante las aporías derivadas de la extensión ilegitima del pensamiento particular al ámbito de la cosmopsicovisión universal. De ahí suele provenir el planteamiento de falsos problemas, el empleo de métodos equívocos y la confusión en las significaciones conceptuales. También la insuficiencia general que se observa en el lenguaje como vehículo de conocimiento, además de los errores constatados desde la antigüedad por la lógica formal en los sofismas, falacias, antinomias, paralogismos y demás modalidades del error "dialéctico". De todo ello pretende liberarse la investigación contemporánea inaugurando sus trabajos con un análisis semántico, sintáctico, semiótico y sintagmático del lenguaje; en él se pone de relieve hasta qué grado puede extenderse la crítica del conocimiento y, por consiguiente, el entredicho para constituir a la filosofía como ciencia, en la primera, elemental y universal acepción, o sea llanamente como conocimiento.

Veamos ahora el segundo concepto, concerniente a la ciencia como conocimiento sistemático. Señalemos a tal respecto que todo conocimiento sistemático es verdadero, aunque no todo conocimiento verdadero es sistemático; la diferencia estriba en que, además de verdadero, el conocimiento puede ser esporádico, y como tal, no sistemático. Así se observa con frecuencia, en cuyo caso no puede afirmarse que tales conocimientos constituyan una ciencia, aunque sean verdaderos. Partimos pues, de que el conocimiento filosófico, para ser cientifico, debe ser sistemático; en otras palabras, para que la filosofía se justifique como ciencia debe ser, además de verdadera, sistemática. Esta convicción induce a la idea del método conforme al cual se realiza como ciencia. El método general del conocimiento consiste en:

a) Partir del objeto por explicar.

b) Concebir la hipótesis explicativa.

c) Verificar la hipótesis en el objeto.

Este es el esquema general que debe cumplir toda ciencia; la cuestión estriba en determinar qué caracteres especificos concurren a la erección de la filosofía como ciencia, además del mecanismo universal consistente en formular y verificar las hipótesis en el problema respectivo. De cualquier modo, la primera condición para reconocer a la filosofía como ciencia se establece afirmando el concepto llano de ciencia en tanto conocimiento demostrado, a partir del cual se llega a la configuración del sistema orgánico y estructurado mediante el empleo del método correspondiente al problema que maneja. Este procedimiento constituye, por lo demás, una tónica general en la filosofía moderna; revierte como norma sustantiva del trabajo académico en la exigencia de apoyar a la investigación en un basamento autónomo, objetivo y progresivo.

La constitución de la ciencia es un proceso paulatino donde se registran 
diversas modalidades y gradaciones; a tal efecto distinguimos la etapa preparatoria del conocimiento cientifico propiamente dicho, el cual designaremos como conocimiento precientifico, de otra etapa que constituye la culminación del saber mediante la autoconciencia lógica, epistemológica y metodológica de sus procedimientos, que llamaremos conocimiento metacientífico. La misma secuela evolutiva se registra en nuestra disciplina; así como existe un conocimiento precientífico integrado por los enunciados que entrañan conocimientos verdaderos, aunque no sistematizados, existe también la que vamos a denominar filosofia precientifica; en ella se incluyen casos similares de ejercicio cognitivo que pueden recibir la atribución filosófica en cuanto afocan determinadas cuestiones comúnmente adjudicadas al filosofar, pero no lo hacen de manera plenamente consciente, o lo que equivale, autoconsciente. Otra cosa se observa en el conocimiento científico y metacientifico; ambos casos entrañan la conciencia de sistematicidad y organizan el cúmulo de problemas que continuamente asaltan a la razón filosófica; se trata de planteamientos inmersos en una suerte de filosofar incidental que la conseja popular ha llamado irónicamente sabiduría confusa, profusa y difusa, como en efecto resulta esta sapiencia pragmática volcada en la vida cotidiana y envuelta en las confusiones que todos sabemos. Inmersos en la multiplicidad de la experiencia, semejantes atributos resultan inherentes al proceso integrativo del saber, pero ello no autoriza a considerarlos como deleznables, sino concomitantes a las primeras etapas del saber, asi en la filosofia como en la ciencia y la vida común.

El concepto de ciencia involucra, pues, dos acepciones básicas: una amplia, equivalente en términos generales a conocimiento, y otra restringida, que avala únicamente al conocimiento sistemático, es decir, a las ciencias propiamente dichas. Ambas dimensiones participan del común denominador que significa la ciencia como saber; a su vez, el saber es definible como la explicación de un objeto planteado en el problema por determinar, lo cual atañe de preferencia al conocimiento científico, aunque esta definición se verifica genéricamente en ambos casos; el conocimiento es siempre scientia, pero el segundo caso comporta el concepto estricto del saber como ciencia, equivalente a disciplina sistemática organizada en un cuerpo doctrinario.

Desde un punto de vista funcional, conocimiento equivale a despejar la incógnita planteada en la ecuación epistémica; el despeje se obtiene mediante el establecimiento de juicios, que a su vez comportan estructuralmente la relación de conceptos. De ahí la definición básica expresada en términos de predicación lógica y de fundamentación axiomática: el conocimiento consiste en la posibilidad de establecer relaciones conceptuales. La explicación del conocimiento y la manera como lleva a cabo su función, constituye el conocimiento del conocimiento, o sea la conciencia trascendental, autoconciencia de formas predicativas y relaciones estructurales en la determinación del saber. 
Ahora bien, retomando el origen del problema, esta definición resulta aplicable a Ia filosofía como un conocimiento que establece relaciones interjudicativas, de análoga manera a como sucede en cualquier rama epistémica. Éste es el requisito genérico que cumple la ciencia en tanto conocimiento; resta por verificar la condición específica donde se estipula a la ciencia como conocimiento sistemático desarrollado en una estructura orgánica. Por ello, al plantear la exigencia de que la filosofía se constituya no sólo como ciencia lato sensu sino como disciplina científica, y en último análisis como ciencia rigurosa, es necesario tener en cuenta que existen ambos conceptos; generalmente cuando hablamos de ciencia se entiende únicamente el segundo, restringido al conocimiento sistemático, y se soslaya el primero, que atañe a la veracidad del saber como un dato objetivamente válido $e$ imbrica en términos generales la problemática inherente a la doctrina de la verdad; ésta es debatida por la gnoseología genética y la epistemología teorética, o sean los dos hemisferios que comprende la teoría del conocimiento. Obviamente, sólo el segundo satisface en plenitud el requisito de cientificidad, desde el momento que verifica la condición erectiva del saber estructurado en un sistema orgánico; el primero contiene la potencialidad parcial del proceso gnoseológico y cobra realidad integrativa en el segundo, de manera que aquél actúa como condición preliminar pero no primordial de éste; sería incongruente segregar al conocimiento fragmentario de la función integrativa que desempeña en 'el sistema, soslayando con ello el valor esencial que comporta como conocimien. to verdadero y prelación antelativa del conocimiento sistemático.

\section{III}

La constitución de la filosofía como ciencia no debería presentar mayor problema si aceptamos previamente que es capaz de erigirse en conocimiento verdadero; sin embargo, aun esta condición elemental no es cabalmente cumplida en las ocasiones que registran alguna distorsión en el prospecto metodológico, traducido al esquema condicionante de ser verdadero, demostrable, autónomo, congruente, limitado, relativo, concreto, etc. El origen de este incumplimiento se encuentra, cuando menos parcialmente, en la naturaleza de la filosofía que, en su ingénita tendencia a obtener un concepto universal del mundo y de la vida, suele desbordar los limites señalados por la circunscripción estricta de un objeto de conocimiento. Otro factor muy influyente en esta gnoseología epistémica radica en el soslayo de la experiencia, por lo cual nuestra disciplina se exhibe en gran medida como especulación, o lo que equivale, como abstracción anticientífica. Estos caracteres riñen con el deside. ratum genéricamente epistémico y específicamente científico, a pesar de lo cual predominan a lo largo de la evolución filosófica que, en estas condiciones, cabría señalar cuando menos en cierto modo como una evolución ca- 
rente de las definiciones aporéticas que son distintivas de la integratividad científica.

A mayor abundamiento, la filosofía tropieza con graves obstáculos para justificar su titularidad sobre cualquier problema de orden material; éste es un requisito básico, pues la carencia de un problema especifico impide a cualquier tipo de reflexión que pueda presentarse como ciencia, y así, en más de una ocasión, lo han puesto en tela de juicio los filósofos mismos. La reiterada duda sobre esta posibilidad básica del filosofar denota su origen en cuanto meditación tendiente a la universalidad, o sea la cosmopsicovisión ubicada sobre un problema particular, lo cual genera la agógica insatisfactoriamente resuelta en cuanto a la capacidad de responder a la demanda de una cosmopsicovisión universal, cuyo desenlace ciframos en la metátesis aporética, o sea el traslado de un problema originalmente filosófico y universal al campo de las ciencias particulares.

A través de la historia se contempla reiteradamente este proceso, lo cual pone a la filosofía en entredicho por carecer supuestamente de los recursos metodologicos que puedan configurar el correspondiente sistema cientifico, sobre todo en un prospecto tan amplio como el implicado en la motivación original de nuestra disciplina. Tales recursos, en cambio, se producen con generosidad en el dominio científico, donde se observa el traslado de los problemas materiales, inicialmente albergados en el manto filosófico, al dominio de la experimentación y la sistematización científicas. Así aconteció con el problema del ser, que florece espléndidamente en el sistema de las ciencias naturales; otro tanto sucede con la temática de la sociedad, que ha dado origen a las ciencias sociales; las cuestiones del hombre están insertas en el tronco de las ciencias antropológicas, y así sucesivamente. El proceso metatético ha actuado muchas veces y constituye la tónica permanente en la historia paralela de la filosofía y las ciencias.

A través de su evolución, la filosofía ha ido cediendo a las ciencias particulares los problemas inicialmente admitidos en su seno; la pretensión metafísica de llegar a verdades últimas, eternas, absolutas e inmutables, cede el campo a los planteamientos particulares y se concreta en función de su particularidad, convirtiéndose en objeto de estudio para una o varias ciencias que, por ello mismo, se denominan particulares. De este modo irrumpe un dilema crucial en el deslinde de éste y cualquier otro apotegma del conocimiento, al cual nos permitimos designar con el nombre de dilema aporético. Representa una alternativa que puede expresarse del siguiente modo: si la filosofia repite lo que afirman las ciencias, genera una duplicidad estéril, y si las contradice, pierde frente a ellas. En estas condiciones, la mayor aporía que establecen las coordenadas inquisitivas para legitimar la subsistencia de nuestra disciplina es la siguiente: ïexiste un problema que sea especifico 
de la filosofía y mantenga al mismo tiempo el carácter universal que le corresponde por tradición?

Una respuesta adecuada debería incluir el sentido que asumen las doctrinas contemporáneas como desenlace de la contradicción ingénita señalada en las posturas: cada una de ellas contiene una verdad particular $y$, sin embargo, la presenta como universal. De ahí el grave error heterónomo cometido con frecuencia: atribuir a la filosofía una temática revelada como pertinente en las ciencias particulares. Tal es el origen de la disputa científico-filosófica que pone en crisis la validez de los conocimientos elaborados para su resolución, lo cual repercute en el entredicho de ciertas filosofías que se ven reemplazadas ventajosamente por las ciencias respectivas. Se plantea entonces otro interrogante capital: ¿cuáles son las disciplinas filosóficas que satisfacen la autonomía sistemática de su problema?

Por ahora podemos afirmar que en calidad de tales disciplinas persisten las clásicamente aceptadas como fundamentales: lógica, ética y estética; en ellas se refrenda la trilogía que constituye virtualmente el denominador común de todos los sistemas, derivado de la tríada antropopsíquica establecida sobre las correspondientes virtudes anímicas de razón, voluntad y sentimiento. Para bocetar un esquema sistemático de tales disciplinas habría que agregar la problemática diacrónica representada por la filosofía de la historia y la canónica propedéutica implícita en la historia de la filosofia, así como la asunción autocrítica que se canaliza en la filosofia de la historia de la filosofia, a la cual podemos llamar también metahistoria filosófica o metafilosofía, entendida como autoconciencia del saber implícito en la metodología trascendental y en el sistema autónomo de las posturas críticas que consideran a la filosofía como una metodología en sí misma. Otras disciplinas suelen figurar eventualmente, pero han sido cuestionadas con frecuencia y quizá expulsadas del tronco filosófico para adjudicar su temática específica a las ciencias respectivas, como por ejemplo: la filosofia de la religión, del lenguaje, de la educación, del hombre, de la naturaleza, etc.

El problema que comporta la erección científica de la filosofía nos lleva de este modo a afrontar un dilema tan crucial como es el correspondiente a la existencia o inexistencia, validez o invalidez, de algunas disciplinas tenidas como filosóficas, y en última instancia la duda se extiende a la integridad de la filosofía misma. Esta dubitación puede proyectarse en las tres o cuatro grandes ramas que hasta ahora se exhiben como puntales inconmovibles del filosofar: lógica, ética y estética -incluyendo en la primera a la epistemología - cuya conjunción está determinada en la filosofía de la historia, entendida como historia de las disciplinas filosóficas. La razón más poderosa que justifica este esquema disciplinario se encuentra en la elocuente circunstancia de que las ciencias particulares acometen cada día con mayor eficacia la excogitación de los valores titulares de las disciplinas fundamentales: ver- 
dad, bondad y belleza, cuyo denominador común engloba la totalidad de los valores.

Con esto llegamos a uno de los polos en que está cifrada la existencia de la filosofía y, por ello mismo, la posibilidad de constituirse como ciencia; creemos que a tal respecto es posible una doble solución aporética: por el lado material se constituye en axiologia, y por el formal, en metodología. El primero recae, sin embargo, en la posibilidad metatética constatada en las doctrinas materiales, a cuyo motivo cabe señalar que las ciencias ofrecen cada día mayores aportaciones en relación al problema axiológico; cosa distinta sucede en el aspecto formal, donde la crisis se resuelve favorablemente, pues al pretender constituirse como ciencia, la filosofía transmuta su vieja naturaleza de concepción universal y pretendidamente material, para erigirse en una nueva ciencia, también universal pero no material, desde el momento que reviste fundamentalmente un carácter formal o metodológico.

Las limitaciones del conocimiento filosófico son, en el aspecto material, cada vez más estrecha; pero no sucede otro tanto en el formal, donde se registra la posibilidad de asumir una función metodológica, para la cual se amplían constantemente sus posibilidades. Por ello reconocemos la autonomía del problema metodológico entre los fundamentales de la filosofía, y no faltan quienes pretenden reducir su destino a metodología, lo cual sucede parcialmente en diversas ocasiones con la postulación formal que preconiza el carácter prioritario de la metodología científica, paralelamente a una metodología cultural, insertas ambas en la concomitante metodología filosófica.

Situados en la coyuntura de justificar un problema que, además de exclusivo sea universal, parece difícil encontrar otro distinto del que denota la metodologia, el cual induce a un nuevo concepto de la filosofía formal; es importante considerar también la aplicación filosófica al problema material de los valores, situado en contrapolo complementario de una filosofía basada en la doctrina axiológica, como un esfuerzo por conservar en ella alguna problemática material. Debemos confesar, sin embargo, que actualmente resulta controvertible cualquier atribución material de la tarea, e inclusive pudiera ser éste un tema extrafilosófico, teniendo en cuenta que el estudio de los valores culturales también corresponde, cada vez en mayor grado, a las ciencias homólogas: la historia, la sociología, la economía, la antropología y demás disciplinas de la cultura. No olvidemos que es ella el recipiente universal de los valores, por lo cual dichas ciencias se constituyen respectivamente en historia, sociologia, economía o antropología de los valores, como de hecho se manejan con frecuencia en sus marcos de referencia conceptual. No parece, por consiguiente, que éste pudiera ser el camino indicado para justificar en definitiva el problema universal y privativo de la filosofia, con miras a su inherente justificación científica.

A cambio de ello, permanece la cuestión metodológica en un dominio 
aún no enajenado, de manera que establecer formalmente lo que son o deben ser los valores, equivale a preguntar por las condiciones formales del conocimiento y su aplicación a la vida cultural. Éste es un tema que subsiste como universal y entronca en los lineamientos del consenso criticista que confiere al filósofo la facultad, ya no de concebir y postular idealmente los valores, sino de establecer su función epistémica y regulativa entendida como coordinación estructural o formal de la existencia. Tal es, en síntesis el problema que señalamos para una axiología formal o metodologia de los valores como versión sistemática y cientifica de la filosofía contemporánea.

De este modo creemos cumplir la condición elemental de toda ciencia, consistente en justificar un problema propio; salvamos al mismo tiempo el reparo proveniente de la paulatina extensión observada en el campo de las ciencias culturales, que amplían y concretan cada vez más el sentido de la normatividad teleológica y la electividad axiológica, por cuya virtud es posible especificar científicamente lo que son y deben ser los valores. Para ello es necesario tener en cuenta las indicaciones que en el campo de la conceptuación cultural promueven las ciencias antropológicas, que son también las ciencias del hombre y la cultura; abordan ellas la determinación concreta de las finalidades existenciales que denominamos valores, tanto en el aspecto de realización como en el de justificación, con los hechos y obras originados en sus diversas ramas, atendiendo a los indices realistas que se manifiestan en la trayectoria evolutiva de la vida cultural sin los cuales el planteamiento axiológico no pasa de ser una abstracción. El presupuesto genérico del valor, proyectado directamente al acto de la valoración, está apoyado por el concurso de las ciencias particulares y establece la cientificidad de la axiología formal entendida como metodología filosófica, que a su vez abre la posibilidad de establecer a la filosofía como ciencia en su avatar axiológico-metodológico. Éste es otro de los aspectos básicos que deben considerarse para establecer la cientificidad filosófica en un plano eminentemente formal, evitando su confusión con las ciencias particulares del valor, que son también las ciencias explicativas del hombre y la cultura.

Esta reiterada insistencia en la función formal que atribuimos a la filosofía, obedece -entre otros aspectos- a la metátesis producida en la acepción material de la axiología; sobre ella es necesario llamar poderosamente la atención. Para percatarnos de la amplitud que reviste la metátesis axiológica, veamos lo que sucede en el problema acogido por las disciplinas capitales de la filosofía, como son la lógica, la ética y la estética, cuya indoctrinación ofrece el reducto tradicional para discernir los valores verdad, bondad y belleza.

Desde el punto de vista filosófico, sabemos que la lógica se convierte en teoría de las ciencias y tiende a develar el sentido de la verdad producida en ellas, la ética se manifiesta como una teoría de la conducta observada en 
la moralidad individual y colectiva, mientras la estética se reafirma como una teoría de la belleza realizada en el arte; éste es el tríptico aporético ubicado en el reducto de la problemática clásica inserta en la cultura. Allí está cifrada la mayor esperanza de legitimidad autónoma para el filosofar; sin embargo, nos vemos obligados a concluir que este reducto se pone en entredicho al observar las ciencias particulares que intervienen con creciente intensidad en la determinación objetiva de su temática, abandonando la inveterada acepción especulativa para acreditarse cada vez más como un tratamiento científico de la cultura.

Por ello, aun las tres ramas capitales de la filosofía clásica se colocan en estrecha dependencia con respecto a las ciencias del hombre, principalmente la psicología, la pedagogía, la sociología y la antropología; son ellas las que aportan los materiales empiriológicos para elaborar la cosmopsicovisión científica y cultural referida a su origen humano. Es, pues, evidente la correlación establecida entre filosofía y ciencias particulares ante un problema común discernido entre éstas y aquélla; la virtual dependencia de la primera frente a las segundas resulta inversamente proporcional a como se supone en la tradición para el común efecto del problema abordado en cada caso.

Sin embargo, la dependencia precientífica de la filosofia no disminuye su valor intrínseco, toda vez que permanece en el seno de la correlación funcional inherente a cualquier ciencia con respecto a su propio objeto; en este caso el objeto es puesto en entredicho $y$, por consiguiente, la disciplina que supuestamente lo maneja. El motivo más conspicuo de la crisis que aflora en el concepto científico de la filosofía entendida como axiología, radica en la profunda metátesis que ha venido operando en la función postulatoria de los valores, manifiesta en el notable incremento de las determinaciones empiriológicas con el proporcional detrimento de la especulación. El aspecto material involucrado en su calidad científica queda pendiente de establecer con el método necesario para aceptar genéricamente el conocimiento esporádico, incorporándolo en cada caso a la disciplina derivada de su planteamiento original; esta función intrínseca registra un apreciable volumen de opiniones circulantes en el conocimiento cotidiano y, además de complementar el saber disciplinario de la filosofía y las ciencias particulares, se vincula indisolublemente a ellas como un conocimiento primario y alternativo de carácter axiológico-material y metodológico-formal.

La consecuencia advertida al reconocer ambas especificaciones científicas, radica en la configuración sistemática del saber, e incorpora los conocimientos significados por las ideas, opiniones, creencias, aforismos, lemas, fragmentos, etc., como formas eventuales de un filosofar incidental, cuya asimilación sistemática auspicia el procedimiento metatético anteriormente descrito. Por ello afirmamos que las opiniones casuísticas de la vida cotidiana enuncian conocimientos verdaderos y paracientíficos, aunque no en la misma propor- 
ción se acreditan como un saber científico propiamente dicho, si por tal se entiende, en sentido estricto, el sistemáticamente organizado, que obviamente supera en nivel integrativo a la producción ocasional de las opiniones características a las primeras etapas en cualquier forma del saber. Es necesario, en todo caso, tener en cuenta las incontables modalidades del pensamiento que, aun siendo verdaderas, no corresponden al saber filosófico y científico en la plena acepción que revisten ambos términos, como un conocimiento formal y material, orgánico y sistemático consciente y autoconsciente apodícticamente fundado y metodológicamente dirigido.

\section{IV}

A partir de consideraciones como las anteriores cumplimos el propósito inherente a este ensayo: la caracterización preambular de la filosofía como ciencia. Es necesario diseñar finalmente el concepto general de ciencia y para ello propondremos una definición que la acepta como todo sistema de conocimientos; este concepto desemboca en la indisoluble correlación existente en los tres grandes niveles integrativos del saber cientifico: el problema, el $m \dot{e}$ todo y el sistema. Desde ese punto de vista, resulta asequible una definición como puede ser la siguiente: ciencia es un sistema de conocimientos que explican un problema mediante el método adecuado.

En otro sentido, el carácter genérico de cualquier conocimiento imbrica la condición básica para ser considerado como científico y establece su estructuración orgánica en un sistema epistémico, de suerte que los conocimientos inicialmente captados en la realidad constituyen, por su carácter contingente y empírico, el material para la edificación de una ciencia que, recíprocamente, se constituye en calidad de estructura orgánica, lo cual genera otro enunciado más amplio: ciencia es un conjunto de conocimientos estructurados en una organización sistemática que vincula, explica y resuelve la multiplicidad de la experiencia.

Esta conclusión nos dice también cuáles son los requisitos que debe cumplir un conjunto de conocimientos para constituirse como ciencia. Desde luego, el concepto generalmente aceptado como definición primaria es el que estipula a la ciencia llanamente como sistema de conocimientos. Esta debe ser la definición básica de toda ciencia; en ella se sobrentiende que el objeto es también un problema por determinar, o como se dice en la epistemología crítica, la incógnita por despejar en la ecuación del conocimiento. A su vez, el objeto primario de toda disciplina encarna en el axioma fundamental que la determina como ciencia particular y señala que el estudio del objeto abre la posibilidad para la constitución del sistema. Éste se erige a partir del axioma definitorio de su problema básico y constriñe dialécticamente a la estructura epistémica del saber a partir del objeto conocido, de suerte que el 
contexto doctrinario de una disciplina encarna la respuesta integral otorgada al interrogante planteado en el axioma subyacente a la base de la ciencia misma.

Entiéndase también que hablar de axioma puede significar, y de hecho significa, un conjunto de postulados coimplicantes cuya definición primaria se extiende a la cadena predicativa de la determinación, proseguida en el ejercicio de la metodología dialéctica; sin ella no es posible configurar el contorno aporético del problema establecido inicialmente por el axioma o conjunto de axiomas, el cual quedará difundido en un gran número de interrogantes secundarios que derivan hacia los planteamientos sucesivos de la postulación inicial, de suerte que cada paso predicativo repite a un nivel superior el mismo problema básico en busca de una solución que, al ser obtenida, vuelve a generar un nuevo planteamiento, y así sucesivamente. La metodologia apodíctica señala el punto de arranque en la connotación predicativa del axioma, cuyo primer nivel es postulado en la concatenación dialéctica y conlleva por modo natural la estructuración orgánica de la ciencia. Los planteamientos se repiten a cada paso, dando origen a sendos interrogantes sucesivos que, en conjunto, integran la estructura sistemática del saber.

La función axiomática se aplica a definir cualquier ciencia, desde el momento que fundamenta el sistema epistémico al cual pertenece el axioma, mediante la definición de la unidad estructural en cuyo derredor se incorpora la multiplicidad de conocimientos constitutivos de la disciplina; la función postulatoria es fundamental porque contiene la unidad organizadora y configuradora de la pluralidad epistémica, determinada en cada caso por la definición del objeto que le pertenece. La definición ofrecida en calidad de axioma comporta patente de idea universal y engloba en primer término el requisito de la ciencia como sistema; en cada disciplina el axioma es equivalente, en cuanto acepción universal, a la unidad orgánica de los conocimientos estructurados en torno al problema común, el cual es definido inexcepcionalmente como postulado característico del problema privativo en dicha ciencia. Éste es el caso de la filosofía, cuyo problema radica primordialmente en el conocimiento mismo y constituye el más importante de todos los problemas.

A partir de esta funcionalidad aporética, en la cual se plantea la cuestión autónoma inherente a cada disciplina, se justifica el anhelo científico de la filosofía mediante la incorporación de la facultad metodológica y la estructuración sistemática coligadas en un cuerpo de doctrina, lo cual permite cumplir las condiciones básicas de toda ciencia. Al mismo tiempo nos vemos inducidos a preguntar: ¿qué clase de ciencia es la filosofía? Este problema conlleva un múltiple sentido, pues atañe a las diferentes acepciones registradas en el concepto de ciencia y filosofía; en un primer plano de importancia deben figurar las diversas maneras como se ha intentado fundar el carácter 
sistemático en tanto correlativo del científico; en todo caso, el requisito primordial estriba en aceptar dicho carácter, pues no se concibe ciencia alguna que renuncie a la configuración de sistema, aunque este hecho constituye apenas el punto de partida y genera los numerosos problemas debatidos en el análisis de la cuestión.

En primer término debemos mencionar la tendencia predominante en la actualidad para realizar la idea científico-filosófica bajo una metodología deductiva que verifique la asunción apodíctica proclamada por el racionalismo, ubicando la idea de ciencia como función normativa y autoconsciente del filosofar. La metodología matemática abona las abundosas corrientes logísticas coincidentes en el vértice funcional e integrativo donde se vinculan dialécticamente los parámetros aporéticos y metodológicos inspirados en el modelo deductivo. Éste es el caso más conspicuo de las tendencias que registra actualmente nuestra disciplina; pero, en términos generales, la condicionalidad científica se establece mediante la relación homóloga del problema planteado y el método aplicable a su resolución, concluyendo en el sistema de conocimientos resultante de su propio tratamiento.

En este orden de consideraciones se impone una refundación de la filosofía en paralelo a la dimensión universal del conocimiento científico; para ello es necesario demostrar que cumple satisfactoriamente la triple condicionalidad exigible a toda ciencia:

a).-Condición aporética Posesión de un problema propio, irreductible a los de Ias ciencias particulares.

b).-Condición metodológica. Aplicación de un método homólogo para desarrollar y explicar el problema.

c).-Condición sistemática. Congruencia estructural entre el problema y el método al integrar el sistema.

La correlación anterior estriba, como lo expresa el concepto mismo, en una relación mutua establecida entre problema, método y sistema, teniendo como base tipificante el reconocimiento científico en cada rama del saber. Dicha correlación señala en cada caso una modalidad especial de llevarse a cabo; se presenta en la matemática con un peculiaridad específica, otra distinta en la física, una más se observa en las ciencias sociales, en las antropológicas; lo propio acontece en la filosofía, cuya manera de configurar la correlación epistémica verifica en su problema las verdades obtenidas en la edificación sistemática como resultado de aplicar el método homólogo a su tratamiento.

Esta correlación se funda en la virtud que designamos con el nombre de homologia estructural y consiste en una similitud manifiesta entre el problema, el método y el sistema de cada disciplina, lo cual determina la permeabilidad cognitiva por cuyo efecto es posible que circulen los elementos epistémicos en los tres planos de la correlación mencionados anteriormente: 
problema, método y sistema. La correlación homóloga es el fundamento de la evolución dialéctica observada en el campo del conocimiento, la cultura y la vida, cuya acepción particular constituye la clave para la integración sincrética de las ciencias. Si no existiera esa correlación, el conocimiento no evolucionaría, o, para decirlo claramente, no existiría.

La tesis final de este ensayo consiste en señalar escuetamente la correlación homóloga entre el problema, el método y el sistema integrativos de cada ciencia; este señalamiento, con toda su simplicidad, es tanto más necesario por cuanto no resulta frecuente comprobarlo en la constitución de las disciplinas filosóficas, donde sólo por excepción se constata una precisa homología en los tres niveles estructurales del organismo; suele acontecer que el problema planteado comporte un falso problema, y que el método sea también erróneo, pues lo mismo se aplican procedimientos objetivos o subjetivos, realistas o idealistas, concretos o abstractos, empiristas o racionalistas; el efecto consiguiente se traduce a la configuración del sistema cuyo contrapolo es el no sistema, o sea la carencia de un sistema verdadero, aunque hubiera la apariencia formal y engañosa del sistema.

Una de las expresiones más frecuentes que ofrece esta deficiencia aporética consiste en confundir el planteamiento de un problema con su resolución, de modo que se cree obtener la respuesta a un problema, cuando en realidad se maneja una distinta manera de presentar el mismo problema. Por ejemplo, al afirmar la existencia del alma como explicación de la vida, lo que se hace es reemplazar el concepto fenoménico de vida por el nouménico de alma, pero las connotaciones de la segunda no agregan ningún coeficiente explicativo al contenido de la primera; esto es lo que significa retomar el problema y presentarlo como solución. Tamaño procedimiento es frecuente en la metafísica cuando hipostasía la entidad fenoménica cuya explicación quiere otorgar; así sucede, por ejemplo, con la célebre teoría platónica de las ideas, cuya existencia trascendente se concibe como réplica de las ideas empiriológicas que ocurren fenoménicamente a la vivencia intelectual: Desde el ángulo pertinente a la epistemología este procedimiento constituye un círculo vicioso, y tal vez una petición de principio. Por lo demás, la aceptación de elementos que funcionan explicativamente aunque en verdad presentan nuevos problemas por determinar, se encuentra inclusive en las disciplinas más firmemente establecidas y forma parte de la secuencia metodológica indesligable del conocimiento científico; en cualquier caso, la sucesión alternante problema-resolución-problema constituye el nódulo dialéctico en la metodología de todas las ciencias y se distingue suficientemente de los errores heterónomos a que nos hemos referido.

$\mathrm{Al}$ exponer los requisitos básicos para constituir a la filosofía como ciencia es necesario apuntar también la condición que, por no cumplirse en la mayoría de los casos, constituye un obstáculo para justificarla en la deseable 
calidad científica: se trata del principio metodológico de autonomia, que encarna en esta época y cada vez más un requerimiento insoslayable cuya ausencia explica por qué la filosofía no se ha justificado unívocamente en un nivel científico, pese a los repetidos intentos del pretérito por llevar a cabo este propósito. Abrigamos la convicción de que nuestra disciplina ingresa ahora definitivamente en ese nivel, pero aun así priva la controversia inherente a lo que significa el filosofar científico; gran parte de las atenciones dedicadas al esclarecimiento de la metodologia constituyen una condición previa a la aceptación material de las tesis que le atañen tradicionalmente.

Hasta ahora, lo importante para la filosofía ha consistido en postular una entidad cualquiera confiriéndole un carácter universal para presentarla en calidad de elemento resolutivo en la cosmopsicovisión respectiva; este procedimiento opera en forma dogmática cuando no se aplica la critificación requerida en cada caso, de lo cual contemplamos un similar desarrollo en las diversas respuestas donde se refleja la problemática del ser, ya esté localizada en el ser mismo o en cualquierà de los elementos que alternativamente acogen las pretendidas soluciones al clásico problema de obtener la explicación universal del ser y la existencia. De este modo, las consideraciones sobre la universalidad tética o material del problema filosófico se extienden a la vida, la cultura, la naturaleza, la materia, las ideas, la energía, los átomos, el número, la proporción, los valores, y en general, a todas y cada una de las hipótesis exaltadas como clave explicativa de la experiencia mediante la asunción universal del ser derivando en cada caso a los elementos que configuran el núcleo doctrinario del planteamiento respectivo; en esta reiterada secuencia que presenta sin más a lo empírico como teorético se amparan dogmáticamente los falsos problemas y conducen inevitablemente a los falsos métodos con las falsas soluciones de los que está saturado el catálogo de las doctrinas filosóficas.

Este procedimiento es insubsistente en la actualidad; la sola presentación de un elemento como universal no basta para convencer de lo que sea realmente la resolución del problema; lo más probable es que constituya uno más entre los numerosos temas por explicar mediante el ejercicio de la determinación cientifica. De cualquier modo subsiste el concepto de la universalidad como norma explicativa del ser, pero la aceptamos a título de hipótesis formal y debe concretarse en la homóloga explicación material; el esquema resolutivo se mantiene fundado en la relación de homologia, aunque varie el procedimiento para llevarlo a cabo. Así, en vez de postular una entidad cualquiera como esencia universal de la realidad, procedemos a vincular los diferentes niveles explicativos donde se presenta la secuencia problema-resolución-problema, eslabonando una serie de gradaciones epistémicas originadas en la multiplicidad de la experiencia, entendida lato sensu como planteamiento de objetividad, para culminar no de un modo directo, sino 
a través de la estructura conceptual-judicativa en una universalidad de la idea y los diversos niveles de jerarquía cognitiva, lo cual se consigue mediante el procedimiento de axiomatización que caracteriza a la moderna filosofía científica.

Por ello, hablar de la filosofía como ciencia equivale a señalar el método de axiomatización como procedimiento indefectible para filosofar; la conclusión que obtenemos en este preámbulo consiste en aceptar la necesidad básica de erigir a la filosofía como ciencia y reconocer.tal carácter en la estructura predicativa fundada sobre el método de axiomatización. El objeto de nuestro ensayo ha consistido en llegar a la frontera que separa al preám. bulo de la tarea propiamente dicha, lo cual conduce a la justificación primordial de la filosofía como ciencia; creemos haber aportado algunas consideraciones básicas y esperamos que de ella se desprendan algunas conclusiones para esclarecer' este inquietante problema en la temática de nuestro tiempo.

INSTITUTO DE INVESTIGACIONES Filosóficas

Miguel Bueno

Universidad Nacional Autónoma de México 